\title{
Combined Aortobifemoral and Femoropopliteal Bypass in Management of Extensive Atherosclerosis: A Case Report
}

\author{
Rahman $\mathrm{MM}^{1}$, Khan $\mathrm{OS}^{2}$, Alam $\mathrm{SAM}^{3}$, Adhikary $\mathrm{AB}^{4}$, Aftabuddin $\mathrm{M}^{5}$
}

\begin{abstract}
A 52 years old non diabetic, normotensive man with aortoiliac and femoropopliteal occlusive disease, combined aortobifemoral and femoropopliteal bypass with silver coated expanded polytetra fluoroethylene (ePTFE) graft was performed. His operation was uneventful, postoperative recovery was excellent. His recovery was early and sent home on 14th postoperative day without symptoms.
\end{abstract}

Key Words: Aortobifemoral bypass, Femoropopliteal bypass, Aortoiliac occlusion, Femoropopliteal occlusion.

\section{Introduction}

Combined Aortobifemoral bypass graft and Femoropopliteal bypass graft is occasionally done in our country. Aortobifemoral bypass with Femoropopliteal bypass graft is performed in patient with atherosclerotic disease of aortoiliac and femoropopliteal segments of the arterial tree with extensive symptoms. The Aortobifemoral bypass considered to be most durable of all bypass of the peripheral arterial system ${ }^{1,2,3}$. In this case report we focus on dramatic improvement of symptoms after this complex surgery within two weeks of surgery.

Case Report

A 52 years old man, smoker, nondiabetic, normotensive, presented with intermittent claudication in both leg, more in right leg for 6 months and extensive leg ulcer in right leg for 1 month. On examination, Right leg was ulcerated,

1. Md. Mahbubur Rahman, MBBS, MS

Final Part Student

Department of Cardiac Surgery

Bangabandhu Sheikh Mujib Medical University, Dhaka.

2. Omar Sadeque Khan, MS

Medical Officer

Department of Cardiac Surgery

Bangabandhu Sheikh Mujib Medical University, Dhaka.

3. Saleh Ahmed Nurul Alam, PhD

Associate Professor

Department of Vascular Surgery

National Institute of Cardiac \& Vascular Diseases, Dhaka.

4. Asit Baran Adhikary, PhD

Professor, Department of Cardiac Surgery

Bangabandhu Sheikh Mujib Medical University, Dhaka.

5. Corresponding Author: Md. Aftabuddin, $\mathrm{PhD}$

Professor \& Chairman

Department of Cardiac Surgery

Bangabandhu Sheikh Mujib Medical University, Dhaka. line of demarcation at ankle joint with foul smell and cool. Left lower limb was normal in color and temperature. His pulse was 80 beats/min, regular with normal volume, Blood pressure was $120 / 70 \mathrm{~mm}$ of $\mathrm{Hg}$. The anterior tibial, posterior tibial, popliteal and femoral pulse was non palpable in both lower limbs. All peripheral pulse of both upper limbs is palpable with normal volume. Motor and sensory function of both lower limbs was intact. Other systemic examination reveals normal findings. Duplex study of right and left lower limb showed, 70-80\% occlusion of Aortoiliac segment. Normal venous system of both limbs. Coronary angiogram showed normal coronary arteries, Peripheral angiogram of abdominal aorta and lower limb arterial system showed, total occlusion of both common Iliac artery (CIA), 100\% occlusion of both External Iliac artery (EIA), normal Internal Iliac artery, common femoral artery (CFA) of right side shows $30-40 \%$ stenosis and left side shows $20-30 \%$ stenosis. Superficial femoral artery (SFA) of both side showed 100\% stenosis, Deep femoral artery (DFA) of left side showed 50\% stenosis, DFA of right side was normal, Popliteal artery of both side was normal, anterior tibial artery of both side was diffusely diseased but posterior tibial artery of both side was normal with normal renal artery.

Aortobifemoral bypass with silver coated ePTFE (50 cmx7 $\mathrm{mm}$ ) graft under epidural anesthesia was planned. The patient was placed in supine position. Under Epidural anesthesia, Povidone iodine painting from mid chest to both lower limb, urethral catheterization and sterile drapping was done. Abdomen was opened via midline incision. Abdominal aorta, both external and internal Iliac artery was identified, Proximal control in distal abdominal aorta was taken and systemic heparinization was done. End arterectomy of distal abdominal aorta and right and left CIA was done. Both Right and left CFA, DFA, SFA was explored and distal control in SFA and DFA was taken. Subcutaneous tunneling was made with a tunnelar from both groin to lower abdomen and side to end anastomosis with ePTFE gaft was performed from Aorta to both CFA. It was ensured that there was no twisting or kinking of the graft. Another bypass was done from right distal SFA to Popliteal Artery ( above knee) with RSVG (Reverse saphenous venous graft) graft. Great saphenous vein was harvested from same limb. ATA, PTA of right side found palpable, SPO2 of right and left lower limb was $100 \%$. The wound was closed with the suction drain kept in situ. On 3rd Postoperative day (POD), all drains were removed and oral anticoagulant started. On 10th POD all stitches were removed. His postoperative recovery was uneventful. Within two weeks after surgery his leg ulcer begins to heal and intermittent claudication 
disappeared. He recovered early and discharged for home on 14th postoperative day with an advice of regular dressing and medication and follow up.

\section{Discussion}

We report the case as it was successfully managed by surgery. The choice of treatment of this complex disease depends on patient's Clinical condition, fitness of patient for a major surgery, site of vascular occlusion and Institutional facility. The usual practice is to reconstruct the Aortoiliac segment first, and then, if symptoms persist, add a reconstruction of Femoropopliteal segment ${ }^{1,4,5}$. But here, we had done combined Aortobifemoral and Femoropopliteal by pass, but result was beyond expectation. If one refers only to patency, then Aortobifemoral grafts are very successful, remaining patency of graft for years, it is nearly $90 \%$ of patients. But relief of symptoms is not synonymous with patency and most patients continue to have intermittent claudication ${ }^{6,7,8}$. But our case showed relief of intermittent claudication within two weeks after surgery.

Aortobifemoral bypass graft is time tested and is one of the best technique for repairing aortoiliac occlusive disease. Although unilateral reconstruction of the iliac vessel used to be common, aortobifemoral bypass is currently preferred. The contralateral disease usually progresses and bilateral femoral bypass eliminates future revision of a unilateral bypass. The cumulative patency of femoropopliteal bypass is $80 \%$ at four years 2 but incidence of graft thrombosis and post operative wound infection is very high in this combined operation3. Combined aortobifemoral and femoropopliteal grafts is occasionally done in our country but ultimate recovery from disease and patency of graft is not reported. But this case showed marked recovery from disease within hospital course that may encourage surgeons of our country in near future.

Though success of this type of surgery depends on longterm patency of the graft, but functional and symptomatic improvement of this case was beyond expectation. To get ultimate outcome we need longterm followup.

\section{References}

1. Malone JM, Moore WS, Goldstone j. The natural history of bilateral Aortofemoral bypass grafts for ischemia of lower extremities. Arch Surgery, 1975;110:1300-06.

2. Eidt J, Charlesworth D. Combined aortobifemoral and femoropopliteal bypass in management of patient with extensive atherosclerosis. Ann vasc surg.1986;1:453-60.

3. Benson J. Thomas J, Whelen CA, Franc C, Spencer MD. Combined aortoiliac and femoropopliteal occlusive disease. Annals of surgery, 1966;163:121-30.

4. Salman E, Ozeren M, Kizilkanat KT, Zengin M, Yucel E. Concomitant reconstruction of combined Aortoiliac and Femoropopliteal occlusive disease at a single stage. Gazi Medical journal. 1992;3:1577-81.

5. Royster RS, Lynn R, Mulcare RJ. Combined aortoiliac and femoropopliteal occlusive disease. Surgery. 1976; 143: 949-52.

6. Summer DS, Strandness DE. Aortoiliac reconstruction in patient with combined iliac and superficial femoral arterial occlusion. Surgery. 1978;84:348-55.

7. Wards, AS, Morris- Jones W. The long term result of profundaplasty in femoropopliteal arterial occlusion. Br J surg. 1977;64:365-67.

8. Mozersky DJ, Sumner DS, Strandness D. Long term results of reconstructive aortoiliac surgery. Am surg. 1972; 123: 503-09. 